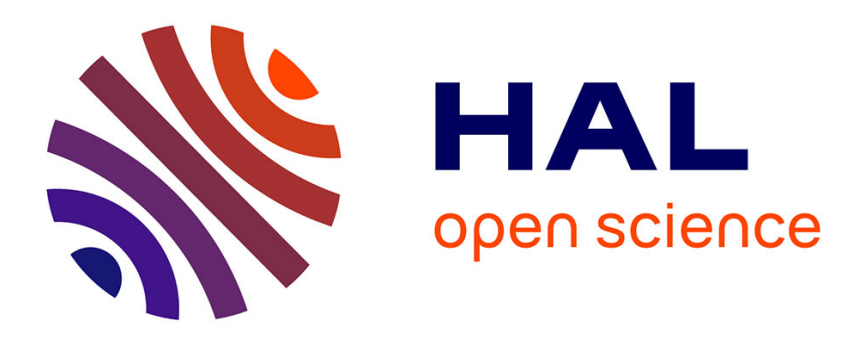

\title{
Essential Supremum with Respect to a Random Partial Order
}

Yuri Kabanov, Emmanuel Lépinette

\section{To cite this version:}

Yuri Kabanov, Emmanuel Lépinette. Essential Supremum with Respect to a Random Partial Order. 2012. hal-00608856v4

\section{HAL Id: hal-00608856 https://hal.science/hal-00608856v4}

Preprint submitted on 22 Jun 2012

HAL is a multi-disciplinary open access archive for the deposit and dissemination of scientific research documents, whether they are published or not. The documents may come from teaching and research institutions in France or abroad, or from public or private research centers.
L'archive ouverte pluridisciplinaire HAL, est destinée au dépôt et à la diffusion de documents scientifiques de niveau recherche, publiés ou non, émanant des établissements d'enseignement et de recherche français ou étrangers, des laboratoires publics ou privés. 


\title{
ESSENTIAL SUPREMUM WITH RESPECT TO A RANDOM PARTIAL ORDER
}

\author{
By YuRI KABANOV and EMmanuel LEPINETTE \\ University of Franche Comté and Paris-Dauphine University \\ Inspired by the theory of financial markets with transaction costs, \\ we study a concept of essential supremum in the framework where \\ a random partial order in $\mathbf{R}^{d}$ is lifted to the space $L^{0}\left(\mathbf{R}^{d}\right)$ of $d$ - \\ dimensional random variables. In contrast to the classical definition, \\ we define the essential supremum as a subset of random variables \\ satisfying some natural properties. An application of the introduced \\ notion to a hedging problem under transaction costs is given.
}

1. Introduction. The aim of this paper is to study a seemingly new concept of essential supremum in the framework where a rather general (possibly, random) partial order in $\mathbf{R}^{d}$ is lifted to the space $L^{0}\left(\mathbf{R}^{d}, \mathcal{F}\right)$ of $d$-dimensional random variables. In contrast to the classical definition of the esssup in $L^{0}=L^{0}(\mathbf{R}, \mathcal{F})$ as a random variable, we define, for $\Gamma \subseteq L^{0}\left(\mathbf{R}^{d}, \mathcal{F}\right)$ and a $\sigma$-algebra $\mathcal{H} \subseteq \mathcal{F}$, the essential supremum, $\mathcal{H}$-Esssup $\Gamma$, as a set of $\mathcal{H}$-measurable random variables satisfying some natural properties. In the classical case, where the partial order in $L^{0}$ is generated by the linear order of the real-line, this set is a singleton, containing the usual essssup when $\mathcal{H}=\mathcal{F}$ and vraimax when $\mathcal{H}$ is trivial.

Our interest to such an object originates from an attempt to give a description of the minimal portfolios dominating a contingent claim in the hedging problem in the presence of proportional transaction costs. In the theory of markets with friction which can be found in the book [2] the value processes are $d$-dimensional adapted processes and contingent claims are $d$-dimensional random variables or processes. Hedging (super-replicating) a European-type contingent claim $Y_{T} \in L\left(\mathbf{R}^{d}, \mathcal{F}_{T}\right)$ means to find a value process $V=\left(V_{t}\right)$ which terminal value dominates the claim in the sense that the difference $V_{T}-Y_{T}$ belongs to the solvency cone $K_{T}$ assumed in this paper to be proper (that is, the model considered here is with the so-called "efficient friction" condition). In other words, $V_{T}$ dominates $Y_{T}$ in the sense of the partial order generated by the cone $K_{T}$. The solvency cone is a fundamental notion of the theory giving a geometric description of the vectors of

AMS 2000 subject classifications: 60G44, G11-G13

Keywords and phrases: Random partial order, Essential supremum, Optimization, Transaction costs, Hedging theorem. 
investor's positions that can be converted (paying the transaction costs) into vectors with non-negative components. In general, solvency may depend on time $t$ as well as on the state of the nature $\omega$ and this is always the case if one considers the representation in terms of physical units.

The language of partial orders induced by the solvency cones fits well to the arbitrage theory of financial markets with transaction costs developed in [2]. Though in the existent models of financial markets the ordering cones $K_{t}(\omega)$ are polyhedral, mathematically it is quite reasonable to consider more abstract models, e.g., those where $\left(K_{t}\right)$ is an adapted set-valued process which values are closed convex cones.

The value (portfolio) process $V$ is called minimal if at the terminal date $V_{T}=Y_{T}$ and any value process $W$ terminating at $Y_{T}$ and dominated by $V$ (i.e. such that $V_{t}-W_{t} \in K_{t}$ for all $t$ ) coincides with $V$. The problem of interest is whether the minimal portfolios do exist and how they can be found. We provide a description of the set of minimal portfolios as the solution of (backward) recursive inclusions involving Esssup. In this context natural questions arise: when Esssup does exist and when it is a singleton? It happened that the natural framework for these questions is much more general than that of models with transaction costs, namely, that of the theory of partial orders.

The main technical hypothesis we use in this paper is the existence of countable multi-utility representation (in the terminology of [1]). To keep the presentation readable we do not work here at full generality: our goal is to fix ideas and built a platform for further studies. Note that the classical concept of essential supremum for scalar random variables is of frequent use in optimal stopping and hedging problems for frictionless market (Snell envelopes, optional decomposition) and extensions of several important results to a multidimensional framework is still pending.

The structure of the paper is the following. In Section 2 we consider a purely deterministic setting. We give the definition of $\operatorname{Sup} \Gamma$ for $\Gamma \in \mathbf{R}^{d}$ and prove a theorem giving a sufficient condition when it is a non-empty set. In the case, where the partial order is generated by a convex closed proper cone this condition is simply boundedness of $\Gamma$ from above in the sense of partial order. In Section 3 we define Esssup $\Gamma$ for $\Gamma \in L^{0}\left(\mathbf{R}^{d}\right)$ and establish its numerous properties. In particular, we are interested in conditions when it is a singleton. In Section 4 we consider a bit more specific model where the random partial order is given by a random cone. In Section 5 we give an application to the hedging problem for European contingent claims under transaction costs.

imsart ver. 2011/05/20 file: Essup-22-06.tex date: June 22, 2012 


\section{Supremum with Respect to a Partial Order in $\mathbf{R}^{d}$.}

\subsection{Basic Concepts.}

We start with some basic concepts and notations restricting ourselves to the Euclidean space $\mathbf{R}^{d}$.

Let $\succeq$ be a partial order in $\mathbf{R}^{d}$, i.e. a binary relation between certain its elements, which is reflexive $(x \succeq x$ ), transitive (if $x \succeq y$ and $y \succeq z$ then $x \succeq z$ ) and antisymmetric (if $x \succeq y$ and $y \succeq x$ then $x=y$ ).

Define an order interval $[x, y]:=\left\{z \in \mathbf{R}^{d}: y \succeq z \succeq x\right\}$ and extend naturally the notation by putting

$$
]-\infty, x]:=\left\{z \in \mathbf{R}^{d}: x \succeq z\right\}, \quad\left[x, \infty\left[:=\left\{z \in \mathbf{R}^{d}: z \succeq x\right\} .\right.\right.
$$

The notations $\Gamma \succeq x$, where $\Gamma$ is a set, means that $y \succeq x$ for all $y \in \Gamma$. In the same spirit: $\Gamma_{1} \succeq \Gamma$ means that $x \succeq y$ for all $x \in \Gamma_{1}$ and $y \in \Gamma$; $\left[\Gamma, \infty\left[:=\cap_{x \in \Gamma}[x, \infty)\right.\right.$ etc. Sometimes we shall use the notation $x \preceq z$ instead of $z \succeq x$.

A partial order is upper semi-continuous (respectively, lower semi-continuous) if $[x, \infty[$ (respectively, $]-\infty, x])$ is closed for any $x \in \mathbf{R}^{d}$ and semicontinuous if it is both upper and lower semi-continuous. Finally, it is called continuous if its graph $\{(x, y): y \succeq x\}$ is a closed subset of $\mathbf{R}^{d} \times \mathbf{R}^{d}$.

We say that a set $\mathcal{U}$ of real-valued functions defined on $\mathbf{R}^{d}$ represents the partial order $\succeq$ if for any $x, y \in \mathbf{R}^{d}$,

$$
x \succeq y \Leftrightarrow u(x) \geq u(y) \quad \forall u \in \mathcal{U} .
$$

This set $\mathcal{U}$ is called multi-utility representation of the partial order. If its elements are continuous functions, we say that $\mathcal{U}$ is a continuous multi-utility representation of the partial order.

Clearly, any partial order can be represented by the family of indicator functions $\mathcal{U}:=\left\{I_{[x, \infty[,}, x \in \mathbf{R}^{d}\right\}$.

The following statement follows from a more general result due to Evren and Ok: any continuous partial order admits a continuous multi-utility representation (see [1], Th. 1).

Note that an arbitrary family $\mathcal{U}$ defines a partial order if the equalities $u(x)=u(y)$ for all $u \in \mathcal{U}$ imply that $x=y$.

The object of our main interest is given by the following

Definition 2.1. Let $\Gamma$ be a non-empty subset of $\mathbf{R}^{d}$ and let $\succeq$ be a partial order. We denote by $\operatorname{Sup} \Gamma$ a subset $\hat{\Gamma}$ of $\mathbf{R}^{d}$ such that the following conditions hold:

imsart ver. 2011/05/20 file: Essup-22-06.tex date: June 22, 2012 
$\left(a_{0}\right) \hat{\Gamma} \succeq \Gamma ;$

$\left(b_{0}\right)$ if $x \succeq \Gamma$, then there is $\hat{x} \in \hat{\Gamma}$ such that $x \succeq \hat{x}$;

$\left(c_{0}\right)$ if $\hat{x}_{1}, \hat{x}_{2} \in \hat{\Gamma}$, then $\hat{x}_{1} \succeq \hat{x}_{2}$ implies $\hat{x}_{1}=\hat{x}_{2}$.

REMARK 2.2. Such a set $\hat{\Gamma}$ is necessarily unique as shown in Lemma 3.3.

The following lemma follows immediately from the continuity assumption.

Lemma 2.3. Let $\succeq$ be a partial order represented by a family of continuous functions. Let $\left(x_{n}\right)$ and $\left(y_{n}\right)$ be two sequences of $\mathbf{R}^{d}$ such that $y_{n} \succeq x_{n}$ for all $n$. Suppose that these sequences converge, respectively, to $x_{\infty}$ and $y_{\infty}$. Then

$$
\bigcap_{n}\left[x_{n}, y_{n}\right] \subseteq\left[x_{\infty}, y_{\infty}\right]
$$

TheOREM 2.4. Let $\succeq$ be a partial order represented by a countable family of continuous functions and such that all order intervals $[x, y], y \succeq x$, are compacts. If the subset $\Gamma$ is such that $\bar{x} \succeq \Gamma$ for some $\bar{x}$, then $\operatorname{Sup} \Gamma \neq \emptyset$. Moreover, if $\Gamma$ is totally ordered, then Sup $\Gamma$ is a singleton formed by a limit point of $\Gamma$.

Proof. Let $\mathcal{U}=\left\{u_{j}\right\}_{j \geq 1}$ be a representing family and fix $x_{0} \in \Gamma$. Without loss of generality we may assume that $\left|u_{j}\right| \leq 1$. We define the function $u()=.\sum 2^{-j} u_{j}($.$) . Observe that this function is continuous. Put a(x):=$ $\inf _{y \in[\Gamma, x]} u(y)$. Since $[\Gamma, x] \subseteq\left[x_{0}, x\right]$, the value $a(x) \geq u\left(x_{0}\right)$. Let $y_{n} \in[\Gamma, x]$ be a sequence such that $u\left(y_{n}\right) \rightarrow a(x)$. Since $[\Gamma, x]$, being an intersection of compacts, is also a compact, we may assume, passing to a subsequence, that $y_{n} \rightarrow y_{\infty}$. In virtue of continuity, $a(x)=u\left(y_{\infty}\right)$.

Define $\Lambda(x)$ as the set of all $y_{\infty} \in[\Gamma, x]$ with $u\left(y_{\infty}\right)=a(x)$ and put $\hat{\Gamma}:=\bigcup_{x \succeq \Gamma} \Lambda(x)$. By above the properties $\left(a_{0}\right)$ and $\left(b_{0}\right)$ hold. Let $\hat{x}_{1}, \hat{x}_{2} \in \hat{\Gamma}$, $\hat{x}_{1} \succeq \hat{x}_{2}$. There is $x_{1} \succeq \Gamma$ such that $\hat{x}_{1} \in \Lambda\left(x_{1}\right)$, i.e. $u\left(\hat{x}_{1}\right)=a\left(x_{1}\right)$. By monotonicity, $u\left(\hat{x}_{1}\right) \geq u\left(\hat{x}_{2}\right)$. But $\hat{x}_{2} \in\left[\Gamma, \hat{x}_{1}\right] \subseteq\left[\Gamma, x_{1}\right]$ and, therefore, $u\left(\hat{x}_{2}\right) \geq a\left(x_{1}\right)$, i.e. $u\left(\hat{x}_{1}\right)=u\left(\hat{x}_{2}\right)$. Since $u_{j}\left(\hat{x}_{1}\right) \geq u_{j}\left(\hat{x}_{2}\right)$ for all $j$ we have necessarily that $u_{j}\left(\hat{x}_{1}\right)=u_{j}\left(\hat{x}_{2}\right)$ for all $j$. Therefore, $\hat{x}_{1}=\hat{x}_{2}$ and $\left(c_{0}\right)$ holds.

Finally, suppose that $\Gamma$ is totally ordered. Define $b:=\sup _{x \in \Gamma} u(x)$. There exists $x_{n} \in \Gamma$ such that $u\left(x_{n}\right) \rightarrow b$. Since $\Gamma$ is totally ordered, we can assume without loss of generality that the sequence $\left(x_{n}\right)$ is increasing and satisfy $x_{n} \in\left[x_{0}, \bar{x}\right]$. Arguing as previously, we get that $x_{n} \rightarrow x_{\infty}$ and $b=u\left(x_{\infty}\right)$. Let $y \in \Gamma$. We have two possibilities. If $y \in\left[x_{n^{\prime}}, \bar{x}\right]$, for some subsequence, then we obtain that $y \in\left[x_{\infty}, \bar{x}\right]$ by virtue of Lemma 2.3. It follows that 
$u(y)=u\left(x_{\infty}\right)$ hence $y=x_{\infty}$. If $y \in \Gamma$ is such that $y \preceq x_{n^{\prime}}$ for some infinite subsequence $\left(x_{n^{\prime}}\right)$ then $y \preceq x_{\infty}$. We conclude that $\operatorname{Sup} \Gamma=\left\{x_{\infty}\right\}$.

REMARK 2.5. It is easily seen that the result holds (with the same proof) not only for $\mathbf{R}^{d}$ but for any topological space such that the order intervals are sequentially compact.

Proposition 2.6. Suppose that the partial order is given by a countable family $\mathcal{U}=\left\{u_{j}\right\}_{j \geq 1}$ of continuous homogeneous functions. Then all the order intervals are compacts.

Proof. Let us consider an arbitrary order interval $[x, z], z \succeq x$, and let $y_{n} \in[x, z]$ be such that $\left|y_{n}\right| \rightarrow \infty$. Passing to a subsequence we may assume that a sequence $\tilde{y}_{n}:=y_{n} /\left|y_{n}\right|$ converges to a point $\tilde{y}_{\infty}$ with $\left|\tilde{y}_{\infty}\right|=1$. Due to the homogeneity, $x /\left|y_{n}\right| \succeq \tilde{y}_{n} \succeq z /\left|y_{n}\right|$ and, therefore,

$$
u_{j}\left(x /\left|y_{n}\right|\right) \geq u_{j}\left(\tilde{y}_{n}\right) \geq u_{j}\left(z /\left|y_{n}\right|\right), \quad j \geq 1 .
$$

Taking the limit, we obtain that $u_{j}\left(\tilde{y}_{\infty}\right)=0$ for all $j$, i.e. $\tilde{y}_{\infty}=0$. A contradiction. So, the order interval $[x, z]$ is bounded, hence, compact.

\subsection{Partial Order in $\mathbf{R}^{d}$ Defined by a Cone.}

In this paper oriented towards financial applications we are interested mainly by the partial order defined by a closed proper convex cone $G \subseteq \mathbf{R}^{d}$. In this case, the relation $x \succeq 0$ means that $x \in G$, and $y \succeq x$ means that $y-x \succeq 0$, i.e. $y \in x+G$. Obviously, it is homogeneous: $y \succeq v$ implies that $\lambda y \succeq \lambda x$ for any $\lambda \geq 0$. Also if $y \succeq x, v \succeq u$, then $x+v \succeq y+u$. The order intervals $[x, \infty[=x+G$ and $]-\infty, x]=x-G$ are closed. Hence, the partial order is semicontinuous. In fact, it is continuous since its graph $\{(x, y): y-x \in G\}$ is a closed subset of $\mathbf{R}^{d} \times \mathbf{R}^{d}$.

By the classical separation theorem the cone $G$ is the intersection of the family of closed half-spaces $L=\left\{x \in \mathbf{R}^{d}: l x \geq 0\right\}$ containing $G$. Its complement $G^{c}$ is the union of the open half-spaces $L^{c}$. In $\mathbf{R}^{d}$ any covering of an open set contains a countable subcovering. Hence, there exists a countable family of vectors $l_{j}$ such that $G=\cap_{j}\left\{x \in \mathbf{R}^{d}: l_{j} x \geq 0\right\}$. It follows that a countable family of linear functions $u_{j}(x)=l_{j} x$ represents the partial order defined by $G$. So, the partial order defined by a closed proper convex cone $G \subseteq \mathbf{R}^{d}$ can be generated by a countable family of linear functions. Clearly, the converse is true.

For the partial order given by a cone $G$ the properties defining the set $\hat{\Gamma}=\operatorname{Sup} \Gamma$ can be reformulated in geometric terms as follows: 
$\left(a_{0}^{\prime}\right) \hat{\Gamma}-\Gamma \subseteq G$

$\left(b_{0}^{\prime}\right)$ if $x-\Gamma \subseteq G$, then there is $\hat{x} \in \hat{\Gamma}$ such that $x-\hat{x} \in G$;

$\left(c_{0}^{\prime}\right)$ if $\hat{x}_{1}, \hat{x}_{2} \in \hat{\Gamma}$, then $\hat{x}_{1}-\hat{x}_{2} \notin G \backslash\{0\}$.

Notation. To distinguish partial orders generated by various cones (a typical situation in financial application) we shall use sometimes the notation $\succeq_{G}$.

As corollary of Theorem 2.4 we have the following result:

THEOREM 2.7. Let $\succeq$ be the partial order generated by a closed proper convex cone $G \subseteq \mathbf{R}^{d}$. If $\Gamma \subseteq \mathbf{R}^{d}$ is such that $\bar{x} \succeq \Gamma$ (i.e. $\bar{x}-\Gamma \subseteq G$ ) for some $\bar{x} \in \mathbf{R}^{d}$, then $\operatorname{Sup} \Gamma \neq \emptyset$.

REMARK 2.8. Any subset $G \subset \mathbf{R}^{d}$ with $G+G \subseteq G$ and $G \cap(-G)=\{0\}$ allows us to define a partial order by putting $x \succeq y$ if $x-y \in G$. Supremum of sets for such partial orders may have rather exotic features. For example, let $d=2$ and

$$
G:=\{(0,0)\} \cup\left\{(x, y) \in \mathbf{Z}_{+}^{2}: x+y \geq 2\right\} .
$$

Under the corresponding partial order, for the set $\Gamma:=\{(0,0),(4,-1)\}$ consisting from two points we have $\operatorname{Sup} \Gamma=\{(4,1) ;(4,2) ;(5,0) ;(5,1) ;(6,0)\}$.

REMARK 2.9. In the literature one can find also other definitions of supremum for partial order. E.g., in the book by Löhne [3] there is a definition adapted to the needs of the vector optimization theory. For the case of $\mathbf{R}^{d}$ with the partial order given by a convex cone $G \neq \mathbf{R}^{d}$ with non-empty interior it can be described as follows. First, it is defined the lower closure of $A$ as the set

$$
\mathrm{Cl}_{-} A:=\{x \in A: x-\operatorname{int} G \subseteq A-\operatorname{int} G\}
$$

and the set of weak maximal points of $A$

$$
\text { wMax } A:=\{x \in A:(x+\operatorname{int} G) \cap A=\emptyset\} .
$$

Finally, $\operatorname{Sup}_{\mathrm{w}} A:=\mathrm{wMax}_{\mathrm{Cl}} A$. For the cone $G=\mathbf{R}_{+}^{d}$ and $A=-\mathbf{R}^{d}$ we have that $\mathrm{Cl}_{-} A=A$ and $\operatorname{Sup}_{\mathrm{w}} A={ }_{\mathrm{wMax}} A=-\partial \mathbf{R}_{+}^{d}$. According to our definition $\operatorname{Sup} A=\{0\}$.

\section{Essential Supremum in $L^{0}\left(\mathbf{R}^{d}\right)$.}


3.1. Setting.

Let $(\Omega, \mathcal{F}, P)$ be a probability space. Let $\mathcal{H}$ be a sub- $\sigma$-algebra of $\mathcal{F}$. We consider in the space $L^{0}\left(\mathbf{R}^{d}, \mathcal{F}\right)$ of $d$-dimensional random variables a partial order defined by a countable family $\mathcal{U}=\left\{u_{j}: j=1,2, \ldots\right\}$ of functions $u_{j}: \Omega \times \mathbf{R}^{d} \rightarrow \mathbf{R}$ with the following properties:

(i) $u_{j}(., x) \in L^{0}(\mathbf{R}, \mathcal{F})$ for every $x \in \mathbf{R}^{d}$;

(ii) $u_{j}(\omega,$.$) is continuous for almost all \omega \in \Omega$.

Namely, for elements $\gamma_{1}, \gamma_{2} \in L^{0}\left(\mathbf{R}^{d}, \mathcal{F}\right)$, the relation $\gamma_{2} \succeq \gamma_{1}$ means that $u_{j}\left(\gamma_{2}\right) \succeq u_{j}\left(\gamma_{1}\right)$ (a.s.) for all $j$.

Definition 3.1. Let $\Gamma$ be a subset of $L^{0}\left(\mathbf{R}^{d}, \mathcal{F}\right)$. We denote by $\mathcal{H}$ Esssup $\Gamma$ a subset $\hat{\Gamma}$ of $L^{0}\left(\mathbf{R}^{d}, \mathcal{H}\right)$ such that the following conditions hold:

(a) $\hat{\Gamma} \succeq \Gamma$;

(b) if $\gamma \in L^{0}\left(\mathbf{R}^{d}, \mathcal{H}\right)$ and $\gamma \succeq \Gamma$, then there is $\hat{\gamma} \in \hat{\Gamma}$ such that $\gamma \succeq \hat{\gamma}$;

(c) if $\hat{\gamma}_{1}, \hat{\gamma}_{2} \in \hat{\Gamma}$, then $\hat{\gamma}_{1} \succeq \hat{\gamma}_{2}$ implies $\hat{\gamma}_{1}=\hat{\gamma}_{2}$.

If $\mathcal{H}$-Esssup $\Gamma$ is a singleton, we denote by $\mathcal{H}$-esssup $\Gamma$ its unique element. Since in this section $\mathcal{H}$ is fixed, we shall omit this symbol and write simply Esssup $\Gamma$ and esssup $\Gamma$. When needed, we note $\operatorname{Esssup}^{\mathcal{U}} \Gamma$ where $\mathcal{U}$ is the family of functions representing the partial order.

We define $\operatorname{Essinf}^{\mathcal{U}} \Gamma:=\operatorname{Esssup}^{-\mathcal{U}}(\Gamma)$ and $\operatorname{essinf}^{\mathcal{U}} \Gamma:=\operatorname{esssup}^{-\mathcal{U}}(\Gamma)$.

Note that for every $\omega$ (except a null set) the countable family of functions $\left\{u_{j}(\omega,).\right\}$ defines a partial order in $\mathbf{R}^{d}$. In the sequel we associate with an order interval $\left[\gamma_{1}, \gamma_{2}\right]$ in $L^{0}\left(\mathbf{R}^{d}, \mathcal{F}\right)$ the order intervals $\left[\gamma_{1}(\omega), \gamma_{2}(\omega)\right]$ in $\mathbf{R}^{d}$ corresponding to these families.

REMARK 3.2. Let $d=1$ and $\succeq$ is the usual total order on the real line. Then $\mathcal{F}$-Esssup $\Gamma=\{\mathcal{F}$-esssup $\Gamma\}$ where $\mathcal{F}$-esssup $\Gamma$ is the classical essential supremum of $\Gamma$. Also in the scalar case, if $\Gamma=\{\xi\}$ and the $\sigma$-algebra $\mathcal{H}$ is trivial, then $\mathcal{H}$-Esssup $\Gamma=\{$ vraimax $\xi\}$.

\subsection{Elementary Properties.}

In this subsection, we consider a partial order $\succeq$ in $L^{0}\left(\mathbf{R}^{d}, \mathcal{F}\right)$ represented by a countable family of functions satisfying (i), (ii).

Lemma 3.3. The set Esssup $\Gamma$ is uniquely defined. 
Proof. Let us consider two subsets $\hat{\Gamma}_{1}$ and $\hat{\Gamma}_{2}$ satisfying (a)-(c). Consider $\hat{\gamma}_{1} \in \hat{\Gamma}_{1}$. Since $\hat{\gamma}_{1}$ dominates the set $\Gamma$, there exists $\hat{\gamma}_{2} \in \hat{\Gamma}_{2}$ with $\hat{\gamma}_{1} \succeq \hat{\gamma}_{2}$. Similarly, there exists $\hat{\gamma}_{1}^{\prime} \in \hat{\Gamma}_{1}$ satisfying $\hat{\gamma}_{2} \succeq \hat{\gamma}_{1}^{\prime}$. Then, $\hat{\gamma}_{1} \succeq \hat{\gamma}_{1}^{\prime}$. But (c) implies that $\hat{\gamma}_{1}=\hat{\gamma}_{1}^{\prime}$. Hence, $\hat{\gamma}_{1}=\hat{\gamma}_{1}^{\prime}=\hat{\gamma}_{2}$, i.e. $\hat{\gamma}_{1} \in \hat{\Gamma}_{2}$. So, $\hat{\Gamma}_{1} \subseteq \hat{\Gamma}_{2}$ and, by symmetry, $\hat{\Gamma}_{1}=\hat{\Gamma}_{2}$.

Lemma 3.4. The set Esssup $\Gamma$ is decomposable, i.e. for any $\hat{\gamma}_{1}, \hat{\gamma}_{2} \in$ Esssup $\Gamma$ and $B \in \mathcal{H}$, we have $\hat{\gamma}_{1} I_{B}+\hat{\gamma}_{2} I_{B^{c}} \in \operatorname{Esssup} \Gamma$.

Proof. Let us consider the set $\tilde{\Gamma}:=\operatorname{Esssup} \Gamma \cup\left\{\hat{\gamma}_{1} I_{B}+\hat{\gamma}_{2} I_{B^{c}}\right\}$. This set satisfies (a)-(c). By the previous lemma, $\tilde{\Gamma}=\operatorname{Esssup} \Gamma$.

Lemma 3.5. Let $\succeq$ be a partial order in $L^{0}\left(\mathbf{R}^{d}, \mathcal{F}\right)$ represented by a countable family of linear functions (in x-variable) satisfying (i), (ii). If Esssup $\Gamma$ is neither an empty set nor a singleton, then Esssup $\Gamma$ is infinite.

Proof. Suppose that Esssup $\Gamma=\left\{\hat{\gamma}_{1}, \hat{\gamma}_{2}, \cdots, \hat{\gamma}_{m}\right\}$ where $\hat{\gamma}_{i} \neq \hat{\gamma}_{j}$ if $i \neq$ $j$. The linearity ensures that the random variable $(1 / k) \hat{\gamma}_{1}+(1-1 / k) \hat{\gamma}_{2}$ dominates $\Gamma$ for each $k \in \mathbf{N}$. The property $(b)$ implies the existence of an integer $N_{k} \in\{1, \cdots, m\}$ such that $(1 / k) \hat{\gamma}_{1}+(1-1 / k) \hat{\gamma}_{2} \succeq \hat{\gamma}_{N_{k}}$. Using again the linearity and the property $(c)$ we deduce that the index $N_{k}$ is not equal to 1 or 2 (otherwise, $\hat{\gamma}_{1}=\hat{\gamma}_{2}$ ). Hence, there exists $j \in\{3, \cdots, m\}$ and an infinite subsequence $\left(k^{\prime}\right)$ such that $N_{k^{\prime}}=j$ for all $k^{\prime}$. By letting $k^{\prime}$ tend to infinity, we infer that $\hat{\gamma}_{2} \succeq \hat{\gamma}_{j}$. therefore, by $(c)$, we have $\hat{\gamma}_{2}=\hat{\gamma}_{j}$. A contradiction.

Example. The linearity assumption above is important. Indeed, let us consider the partial order in $\mathbf{R}^{2}$ given by the family $\mathcal{U}=\left\{u_{1}, u_{2}, u_{3}\right\}$, where $u_{1}(x)=a x, u_{2}(x)=b x$ with $a=(1 / \sqrt{2}, 1 / \sqrt{2}), b=(0,1)$, and $u_{3}(x)=|x|$. Let $\Gamma=\{0, p\}$ where $p=(-1 / \sqrt{2},-1 / \sqrt{2})$. Then $x \succeq \Gamma$ if and only if $a x \geq 0, b x \geq 0$, and $|x| \geq 1$. That is $x \in G$ and $|x| \geq 1$, where $G$ is the cone $\{x: a x \geq 0, b x \geq 0\}$. One can easily check that $\operatorname{Sup} \Gamma=\{A, B\}$ where $A=(-1 / \sqrt{2}, 1 / \sqrt{2}), B=(1,0)$, see Figure 5 . 


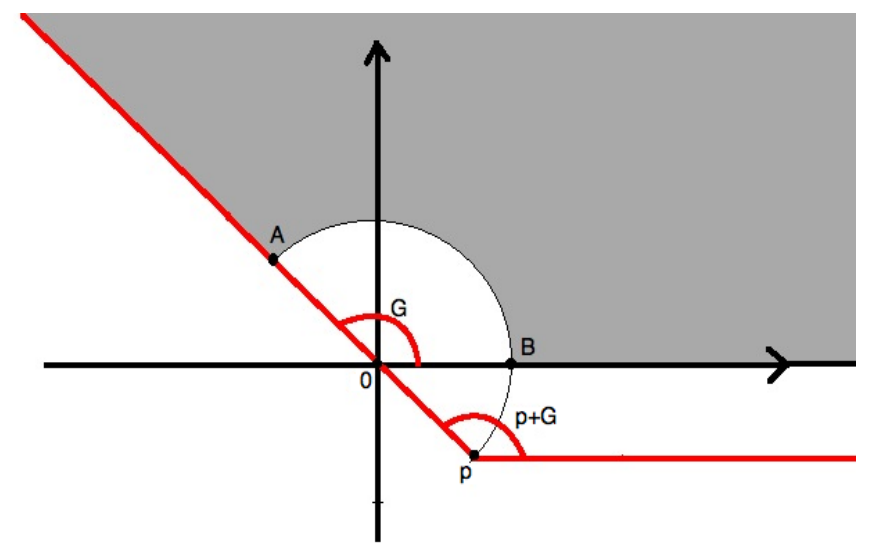

FIG 1. The grey-coloured domain corresponds to the set of all points dominating $\Gamma=\{0, p\}$, hence, $\operatorname{Sup} \Gamma=\{A, B\}$.

Lemma 3.6. Assume that the essential supremum of any finite subset of $L^{0}\left(\mathbf{R}^{d}, \mathcal{F}\right)$ is a singleton. For $\Gamma \subseteq L^{0}\left(\mathbf{R}^{d}, \mathcal{F}\right)$, let the upward completion be defined as

$$
\Gamma^{\mathrm{up}}:=\left\{\operatorname{esssup}\left\{\gamma_{j_{1}}, \cdots, \gamma_{j_{n}}\right\}: \gamma_{j_{k}} \in \Gamma, n \in \mathbf{N}\right\}
$$

Then

$$
\operatorname{Esssup} \Gamma=\operatorname{Esssup} \Gamma^{\mathrm{up}}
$$

Proof. Put $\hat{\Gamma}:=\operatorname{Esssup} \Gamma^{\mathrm{up}}$. Since Esssup $\Gamma^{\mathrm{up}} \succeq \operatorname{esssup}\{\gamma\} \succeq \gamma$ whatever is $\gamma \in \Gamma$, the set $\hat{\Gamma}$ satisfies the property $(a)$. Let $\gamma \in L^{0}\left(\mathbf{R}^{d}, \mathcal{H}\right)$ be such that $\gamma \succeq \Gamma$. In particular, $\gamma$ dominates the elements $\gamma_{j_{k}} \in \Gamma, 1 \leq k \leq n$. By the assumption the essential supremum of the letters is a singleton. Therefore, $\gamma \succeq \Gamma^{\text {up }}$. Thus, there is an element $\hat{\gamma} \in \operatorname{Esssup} \Gamma^{\text {up }}$ such that $\gamma \succeq \hat{\gamma}$. That is $\hat{\Gamma}$ satisfies the property $(b)$. Finally, let $\hat{\gamma}_{1}, \hat{\gamma}_{2} \in \operatorname{Esssup} \Gamma^{u p}$ be such that $\hat{\gamma}_{1} \succeq \hat{\gamma}_{2}$. By definition of Esssup we have that $\hat{\gamma}_{1}$ coincides with $\hat{\gamma}_{2}$ and $(c)$ also holds.

\subsection{Existence.}


TheOREm 3.7. Let $\succeq$ be a partial order in $L^{0}\left(\mathbf{R}^{d}, \mathcal{F}\right)$ represented by a countable family of functions satisfying (i), (ii) and such that all order intervals $\left[\gamma_{1}(\omega), \gamma_{2}(\omega)\right], \gamma_{2} \succeq \gamma_{1}$, are compacts a.s. If a non-empty subset $\Gamma$ is such that $\bar{\gamma} \succeq \Gamma$ for some $\bar{\gamma} \in L^{0}\left(\mathbf{R}^{d}, \mathcal{H}\right)$, then $\mathcal{H}$-Esssup $\Gamma \neq \emptyset$.

Proof. Let $\mathcal{U}=\left\{u_{j}\right\}_{j \geq 1}$ be a representing family. Without loss of generality we may assume that $\left|u_{j}\right| \leq 1$. Put

$$
u(\omega, z):=\sum_{j \geq 1} 2^{-j} u_{j}(\omega, z) .
$$

The function $z \mapsto u(\omega, z)$ is continuous and its absolute value is bounded by unit. Fix arbitrary $\gamma_{0} \in \Gamma$. Take $\gamma \in L^{0}\left(\mathbf{R}^{d}, \mathcal{H}\right)$ such that $\gamma \succeq \Gamma$. For any $\zeta \in L^{0}([\Gamma, \gamma], \mathcal{H})$ the mapping $\omega \mapsto u(\omega, \zeta(\omega))$ is an $\mathcal{F}$-measurable random variable taking values in the interval $[-1,1]$. Put

$$
a(\gamma):=\inf _{\zeta \in L^{0}([\Gamma, \gamma], \mathcal{H})} E u(\zeta)
$$

and consider a sequence $\zeta_{n} \in L^{0}([\Gamma, \gamma], \mathcal{H})$ such that $a(\gamma)=\lim _{n} E u\left(\zeta_{n}\right)$.

Without loss of generality we may assume that the sequence of random variables $u\left(\zeta_{n}\right)$ is such that the conditional expectations $E\left(u\left(\zeta_{n}\right) \mid \mathcal{H}\right)$ are decreasing. Indeed, we can replace the sequence $\zeta_{n}$ by the sequence $\zeta_{n}^{\prime}$ by putting $\zeta_{1}^{\prime}=\gamma$, and defining recursively the random variables

$$
\zeta_{n}^{\prime}:=\zeta_{n-1}^{\prime} I_{\left\{E\left(u\left(\zeta_{n-1}^{\prime}\right) \mid \mathcal{H}\right) \leq E\left(u\left(\zeta_{n}\right) \mid \mathcal{H}\right)\right\}}+\zeta_{n} I_{\left\{E\left(u\left(\zeta_{n-1}^{\prime}\right) \mid \mathcal{H}\right)>E\left(u\left(\zeta_{n}\right) \mid \mathcal{H}\right)\right\}}, \quad n \geq 2 .
$$

Due to the assumption of the theorem, the order intervals $\left[\gamma_{0}(\omega), \gamma(\omega)\right]$ are compact (a.s.). It follows that $\sup _{n}\left|\zeta_{n}\right|<\infty$ a.s. By virtue of the lemma on converging subsequences (Lemma 2.1.2 [2]) there exists a strictly increasing sequence of $\mathcal{H}$-measurable integer-valued random variables $\tau_{k}$ such that the sequence $\zeta_{\tau_{k}}$ converges a.s. to some $\zeta$ such that $\Gamma \preceq \zeta \preceq \gamma$. The monotonicity implies that

$$
E\left(u\left(\zeta_{\tau_{k}}\right) \mid \mathcal{H}\right)=\sum_{m \geq k} E\left(u\left(\zeta_{m}\right) \mid \mathcal{H}\right) I_{\left\{\tau_{k}=m\right\}} \leq E\left(u\left(\zeta_{k}\right) \mid \mathcal{H}\right) .
$$

It follows that

$$
E u\left(\zeta_{\tau_{k}}\right) \leq E u\left(\zeta_{k}\right)
$$

Using the continuity of $u(\omega,$.$) and the Lebesgue theorem on dominated$ convergence we have:

$$
\begin{aligned}
& E u(\zeta)=E \lim _{k} u\left(\zeta_{\tau_{k}}\right) \leq \lim _{k} E u\left(\zeta_{k}\right)=a(\gamma) \\
& \text { imsart ver. 2011/05/20 file: Essup-22-06.tex date: June 22, } 2012
\end{aligned}
$$


Thus,

$$
a(\gamma)=E u(\zeta)
$$

We denote by $\Lambda(\gamma)$ the set of all random variables $\zeta \in L^{0}([\Gamma, \gamma], \mathcal{H})$ verifying (3.2) and define the set

$$
\widehat{\Gamma}:=\bigcup_{\gamma \in L^{0}([\Gamma, \infty[, \mathcal{H})} \Lambda(\gamma) .
$$

It remains to show that this set satisfies $(a)-(c)$. Obviously, $\widehat{\Gamma} \succeq \Gamma$, i.e. $(a)$ holds. If $\zeta \in L^{0}([\Gamma, \infty[, \mathcal{H})$, then $\zeta \succeq \Lambda(\zeta)$ by construction, i.e. $(b)$ holds. At last, consider $\widehat{\zeta}_{1}, \widehat{\zeta}_{2} \in \widehat{\Gamma}$ with $\widehat{\zeta}_{1} \in \Lambda\left(\gamma_{1}\right)$ and $\widehat{\zeta}_{2} \in \Lambda\left(\gamma_{2}\right), \gamma_{1}, \gamma_{2} \in[\Gamma, \infty[$, such that $\widehat{\zeta}_{1} \succeq \widehat{\zeta}_{2}$. Suppose that $\widehat{\zeta}_{1} \neq \widehat{\zeta}_{2}$ and, hence, there is $i$ for which $u_{i}\left(\widehat{\zeta}_{1}\right)-u_{i}\left(\widehat{\zeta}_{2}\right) \geq 0$ and the inequality is strict on a non-null set. It follows that there exists a non-null set $B \in \mathcal{H}$ on which

$$
E\left(u_{i}\left(\widehat{\zeta}_{1}\right)-u_{i}\left(\widehat{\zeta}_{2}\right) \mid \mathcal{H}\right)>0
$$

Observe that for all $j$

$$
u_{j}\left(\widehat{\zeta}_{2} I_{B}+\widehat{\zeta}_{1} I_{B^{c}}\right)=u_{j}\left(\widehat{\zeta}_{2}\right) I_{B}+u_{j}\left(\widehat{\zeta}_{1}\right) I_{B^{c}}
$$

and

$$
E\left(u_{j}\left(\widehat{\zeta}_{2} I_{B}+\widehat{\zeta}_{1} I_{B^{c}}\right) \mid \mathcal{H}\right)=E\left(u_{j}\left(\widehat{\zeta}_{2}\right) \mid \mathcal{H}\right) I_{B}+E\left(u_{j}\left(\widehat{\zeta}_{1}\right) \mid \mathcal{H}\right) I_{B^{c}}
$$

for all $j$. It follows that

$$
a\left(\gamma_{1}\right)=\operatorname{Eu}\left(\widehat{\zeta}_{1}\right)>\operatorname{Eu}\left(\widehat{\zeta}_{2} I_{B}+\widehat{\zeta}_{1} I_{B^{c}}\right)
$$

where $\widehat{\zeta}_{2} I_{B}+\widehat{\zeta}_{1} I_{B^{c}} \in\left[\Gamma, \widehat{\zeta}_{1}\right] \subseteq\left[\Gamma, \gamma_{1}\right]$. This is a contradiction. Hence, $(c)$ also holds.

Lemma 3.8. Let $\succeq$ be a partial order represented by a countable family of functions satisfying (i), (ii) and such that all order intervals $\left[\gamma_{1}(\omega), \gamma_{2}(\omega)\right]$, $\gamma_{2} \succeq \gamma_{1}$, are compacts a.s. Suppose that for any $\hat{\gamma}_{1}, \hat{\gamma}_{2} \in L^{0}\left(\mathbf{R}^{d}, \mathcal{F}\right)$, the set Essinf $\left\{\hat{\gamma}_{1}, \hat{\gamma}_{2}\right\}$ is either singleton or empty. Then, for any $\Gamma \subseteq L^{0}\left(\mathbf{R}^{d}, \mathcal{F}\right)$ the set Esssup $\Gamma$ is either singleton or empty.

Proof. Suppose that some $\Gamma \subseteq L^{0}\left(\mathbf{R}^{d}, \mathcal{F}\right)$ is such that Esssup $\Gamma$ contains two different points $\hat{\gamma}_{1}$ and $\hat{\gamma}_{2}$. By the existence Theorem 3.7 above the set Essinf $\left\{\hat{\gamma}_{1}, \hat{\gamma}_{2}\right\}$ is non-empty and, by the hypothesis, it is a singleton formed by some element $\gamma^{*}$. If $\gamma \in \Gamma$, then $\gamma \preceq\left\{\hat{\gamma}_{1}, \hat{\gamma}_{2}\right\}$ and, therefore, $\gamma \preceq \gamma^{*}$, i.e. $\gamma^{*} \succeq \Gamma$. Hence, there exists $\hat{\gamma} \in \operatorname{Esssup} \Gamma$ such that $\gamma^{*} \succeq \hat{\gamma}$. Therefore, $\left\{\hat{\gamma}_{1}, \hat{\gamma}_{2}\right\} \succeq \hat{\gamma}$ implying that $\hat{\gamma}_{1}=\hat{\gamma}$ and $\hat{\gamma}_{2}=\hat{\gamma}$. A contradiction.

imsart ver. 2011/05/20 file: Essup-22-06.tex date: June 22, 2012 
3.4. More Properties of Esssup.

To relate our result with the classical concept of essential supremum of a set of scalar random variables, it is more convenient to consider the space $L^{0}(\mathbf{R} \cup\{+\infty\}, \mathcal{F})$. The natural partial order in this case can be given by a single function, e.g., $u(x)=x$ or any increasing strictly monotone function; the choice $u(x)=\arctan x$ is convenient since the latter is bounded.

Lemma 3.9. Let $\Gamma \neq \emptyset$ be a subset of $L^{0}(\mathbf{R} \cup\{+\infty\}, \mathcal{F})$. Then $\mathcal{H}$ Esssup $\Gamma$ is a singleton. In particular, $\mathcal{H}$-Esssup $\Gamma=\{\mathcal{H}$-esssup $\Gamma\}$.

Proof. Working with $u(x)=\arctan x$ we observe that the arguments of the previous theorem (with the random variable $\bar{\gamma}$ identically equal to infinity) require no changes. It is easy to see that $a(\gamma)$ does not depend on $\gamma \succeq \Gamma$. Finally, there exists only one element $\zeta \in L^{0}([\Gamma, \infty], \mathcal{H})$ such that $(3.2)$ holds (otherwise we could diminish the value of the right-hand side by $\zeta \wedge \zeta^{\prime}$ ).

REMARK 3.10. We have $\mathcal{H}$-esssup $\Gamma \succeq \mathcal{F}$-esssup $\Gamma$.

Proposition 3.11. Let $\succeq$ be a partial order in $L^{0}\left(\mathbf{R}^{d}\right)$ represented by a countable family $\mathcal{U}$ of functions satisfying (i), (ii). Let $\Gamma \subseteq L^{0}\left(\mathbf{R}^{d}, \mathcal{H}\right)$ be a totally ordered subset such that there exists $\bar{\gamma} \in L^{0}\left(\mathbf{R}^{d}, \mathcal{H}\right)$ with $\Gamma \preceq \bar{\gamma}$. Then Esssup $\Gamma$ is a singleton esssup $\Gamma$. Moreover, there is a strictly increasing sequence of $\mathcal{H}$-measurable integer-valued random variables $\tau_{n}$ such that $\left(\gamma_{\tau_{n}}\right)$ converges increasingly to esssup $\Gamma$ a.s.

Proof. Put $b:=\sup _{\gamma \in \Gamma} E u(\gamma)$ where $u$ is defined above. Take $\gamma_{n} \in \Gamma$ such that $E u\left(\gamma_{n}\right) \uparrow b$. The set $\Gamma$ being totally ordered, we may assume without loss of generality that $\left(\gamma_{n}\right)$ is order increasing. Since $\gamma_{n} \in\left[\gamma_{1}, \bar{\gamma}\right]$, we infer that $\liminf \operatorname{in}_{n}\left|\gamma_{n}\right|<\infty$. In virtue of Lemma 2.1.2 [2] there is a strictly increasing sequence of $\mathcal{H}$-measurable integer-valued random variables $\tau_{n}$ such that $\left(\gamma_{\tau_{n}}\right)$ converges a.s. to some $\hat{\gamma} \preceq \bar{\gamma}$. Recall that

$$
\gamma_{\tau_{n}}=\sum_{m \geq n} \gamma_{m} I_{\left\{\tau_{n}=m\right\}} \in L^{0}\left(\mathbf{R}^{d}, \mathcal{H}\right)
$$

Since both sequences $\left(\gamma_{n}\right)$ and $\left(\tau_{n}\right)$ are increasing, we deduce that the sequence $\left(\gamma_{\tau_{n}}\right)$ is also increasing. As $\tau_{n} \geq n$, we get that $\gamma_{\tau_{m}} \succeq \gamma_{n}$ if $m \geq n$ implying that $\hat{\gamma} \succeq \gamma_{n}$ for every $n$ and, also $b=E u(\hat{\gamma})$. It follows that Esssup $\Gamma=\{\hat{\gamma}\}$. Indeed, if $\gamma \succeq \Gamma$, then $\gamma \succeq\left\{\gamma_{\tau_{n}}: n \in \mathbf{N}\right\}$ and, taking the limit, we get that $\gamma \succeq \hat{\gamma}$, i.e. the singleton $\{\hat{\gamma}\}$ satisfies $(a)-(c)$. 
3.5. Properties of Esssup for Homogeneous Generating Functions.

In this subsection we shall work assuming that the functions defining the partial order are linear (in $x$ variable). For a set $\Gamma \subseteq L^{0}\left(\mathbf{R}^{d}, \mathcal{F}\right)$ and $\lambda \in L^{0}\left(\mathbf{R}_{+}, \mathcal{H}\right)$, we define the set $\lambda \Gamma:=\{\lambda \gamma: \gamma \in \Gamma\}$.

LEMMA 3.12. Let $\succeq$ be a partial order in $L^{0}\left(\mathbf{R}^{d}, \mathcal{F}\right)$ represented by a countable family $\mathcal{U}$ of homogeneous functions satisfying (i), (ii). Let a set $\Gamma \subseteq L^{0}\left(\mathbf{R}^{d}, \mathcal{F}\right)$ be such that $\operatorname{Esssup} \Gamma \neq \emptyset$. If $\lambda \in L^{0}\left(\mathbf{R}_{+}, \mathcal{H}\right)$, then

$$
\operatorname{Esssup}(\lambda \Gamma)=\lambda \operatorname{Esssup} \Gamma \text {. }
$$

Proof. Let $\widehat{\Gamma}_{\lambda}:=\lambda$ Esssup $\Gamma$. Since multiplication on elements of $L^{0}\left(\mathbf{R}_{+}, \mathcal{H}\right)$ preserves the order, we have $\widehat{\Gamma}_{\lambda} \succeq \lambda \Gamma$. Let $\gamma \in L^{0}\left(\mathbf{R}^{d}, \mathcal{H}\right)$ and $\gamma \succeq \lambda \Gamma$. Take an arbitrary element $\tilde{\gamma}_{0} \in$ Esssup $\Gamma$. Then

$$
\gamma_{1}:=\lambda^{-1} \gamma I_{\{\lambda \neq 0\}}+\tilde{\gamma}_{0} I_{\{\lambda=0\}} \succeq \Gamma
$$

and, in virtue of the property (b) for $\Gamma$, there is $\hat{\gamma} \in \operatorname{Esssup} \Gamma$ such that $\gamma_{1} \succeq \hat{\gamma}$. It follows that $\gamma \succeq \lambda \hat{\gamma} \in \widehat{\Gamma}_{\lambda}$. Finally, let $\hat{\gamma}_{1}, \hat{\gamma}_{2} \in \hat{\Gamma}_{\lambda}$ be such that $\hat{\gamma}_{1} \succeq \hat{\gamma}_{2}$. By definition of $\hat{\Gamma}_{\lambda}$ we have that $\hat{\gamma}_{i}=\lambda \tilde{\gamma}_{i}$, where $\tilde{\gamma}_{i} \in \operatorname{Esssup} \Gamma$, $i=1,2$. Also,

$$
\bar{\gamma}_{i}:=\tilde{\gamma}_{i} I_{\{\lambda \neq 0\}}+\tilde{\gamma}_{0} I_{\{\lambda=0\}} \in \operatorname{Esssup} \Gamma .
$$

and $\bar{\gamma}_{1} \succeq \bar{\gamma}_{2}$. The property $(c)$ for $\Gamma$ implies that $\bar{\gamma}_{1}=\bar{\gamma}_{2}$. Hence, $\hat{\gamma}_{1}=\hat{\gamma}_{2}$. Therefore, the set $\Gamma_{\lambda}$ satisfies all the conditions defining $\operatorname{Essup}(\lambda \Gamma)$.

We introduce the following condition:

(iii) There is $\gamma^{0} \in L^{0}\left(\mathbf{R}^{d}, \mathcal{H}\right)$ such that $\left\{\zeta \in L^{0}\left(\mathbf{R}^{d}, \mathcal{F}\right):|\zeta| \leq 1\right\} \preceq \gamma^{0}$.

Proposition 3.13. Let $\succeq$ be a partial order in $L^{0}\left(\mathbf{R}^{d}, \mathcal{F}\right)$ represented by a countable family $\mathcal{U}$ of homogeneous functions satisfying (i), (ii), (iii). Let $\Gamma \subseteq L^{0}\left(\mathbf{R}^{d}, \mathcal{F}\right)$ and $|\Gamma|:=\{|\gamma|: \gamma \in \Gamma\}$. Suppose that $\xi:=\operatorname{esssup}|\Gamma|<\infty$ a.s. Then Esssup $\Gamma$ is not empty.

Proof. Consider $\tilde{\Gamma}:=(1+\xi)^{-1} \Gamma$. By (iii), $\tilde{\Gamma} \preceq \gamma^{0}$ where $\gamma^{0} \in L^{0}\left(\mathbf{R}^{d}, \mathcal{H}\right)$. Applying Theorem 3.7, we deduce that Esssup $\tilde{\Gamma}$ is not empty. It follows that Esssup $\Gamma$ is not empty and is given by $\operatorname{Essup} \Gamma=(1+\xi) \operatorname{Essup} \widetilde{\Gamma}$. This identity follows from Lemma 3.12.

It is easily seen that the above results hold also when all functions of the representing family are (positive) homogeneous of order $\gamma \neq 0$. 
LEMMA 3.14. Let $\succeq$ be a partial order in $L^{0}\left(\mathbf{R}^{d}, \mathcal{F}\right)$ represented by a countable family $\mathcal{U}$ of linear functions satisfying (i), (ii), and (iii) and let $\Gamma \subseteq L^{0}\left(\mathbf{R}^{d}, \mathcal{F}\right)$. Then

$\mathcal{F}$-esssup $|\Gamma|<\infty \Leftrightarrow$ there are $\gamma_{1}, \gamma_{2} \in L^{0}\left(\mathbf{R}^{d}, \mathcal{F}\right)$ such that $\gamma_{1} \preceq \Gamma \preceq \gamma_{2}$.

Proof. $(\Rightarrow)$ By the above proposition there are $\gamma_{1} \in \mathcal{F}$-Essinf $\Gamma \neq \emptyset$ and $\gamma_{2} \in \mathcal{F}$-Esssup $\Gamma \neq \emptyset$ with the needed property.

$(\Leftarrow)$ Suppose that the set $B:=\{\mathcal{F}$-esssup $|\Gamma|=\infty\}$ is non-null. Take a sequence $\gamma^{n} \in \Gamma$ such that $\left|\gamma^{n}\right| \uparrow \mathcal{F}$-esssup $|\Gamma|$. Let us define the random variables $\widetilde{\gamma}^{n}:=\gamma^{n}\left(\left|\gamma^{n}\right|+1\right)^{-1}, \widetilde{\gamma}_{1}^{n}:=\gamma_{1}\left(\left|\gamma^{n}\right|+1\right)^{-1}$, and $\widetilde{\gamma}_{2}^{n}:=\gamma_{2}\left(\left|\gamma^{n}\right|+1\right)^{-1}$. Using Lemma 2.1.2 [2] we may assume that $\widetilde{\gamma}_{n} \rightarrow \widetilde{\gamma}$ with $|\widetilde{\gamma}|=1$ on $B$. On the other hand, in virtue of $(i i)$, we have that $\widetilde{\gamma}_{1}^{n} \preceq \widetilde{\gamma}_{n} \preceq \widetilde{\gamma}_{2}^{n}$ for all $n$. It follows that $\widetilde{\gamma}=0$ on the set $B$. A contradiction.

\section{Essential Supremum in $L^{0}\left(R^{d}\right)$ with Respect to a Random Cone.}

\subsection{Setting.}

Let $(\Omega, \mathcal{F}, P)$ be a complete probability space and let $\omega \mapsto G(\omega) \subseteq \mathbf{R}^{d}$ be a measurable set-valued mapping whose values are closed convex cones. The measurability is understood as the measurability of the graph, i.e. we assume that

$$
\operatorname{graph} G:=\left\{(\omega, x) \in \Omega \times \mathbf{R}^{d}: x \in G(\omega)\right\} \in \mathcal{F} \otimes \mathcal{B}\left(\mathbf{R}^{d}\right) .
$$

The positive dual $G^{*}$ of $G$ is defined as the mapping whose values are closed convex cones

$$
G^{*}(\omega):=\left\{x \in \mathbf{R}^{d}: x y \geq 0, \forall y \in G(\omega)\right\},
$$

where $x y$ is the scalar product. Note that that $0 \in L^{0}(G, \mathcal{F}) \neq \emptyset$.

The fundamental fact of the theory of set-valued analysis is that any measurable mapping whose values are closed subsets admits a Castaing representation (see, e.g. [4]). In our case this means that there exists a countable set of measurable selectors $\xi_{i}$ of $G$ such that $G(\omega)=\overline{\left\{\xi_{i}(\omega): i \in \mathbf{N}\right\}}$ for all $\omega \in \Omega$. Thus,

$$
\operatorname{graph} G^{*}=\left\{(\omega, y) \in \Omega \times \mathbf{R}^{d}: y \xi_{i}(\omega) \geq 0, \forall i \in \mathbf{N}\right\} \in \mathcal{F} \otimes \mathcal{B}\left(\mathbf{R}^{d}\right),
$$

i.e. $G^{*}$ is a measurable mapping and admits a Castaing representation, i.e. there exists a countable set of measurable selectors $\eta_{i}$ of $G^{*}$ such that $G^{*}(\omega)=\overline{\left\{\eta_{i}(\omega): i \in \mathbf{N}\right\}}$ for all $\omega \in \Omega$. Since $G=\left(G^{*}\right)^{*}$,

$$
\begin{aligned}
& \quad G(\omega)=\left\{(\omega, x) \in \Omega \times \mathbf{R}^{d}: \eta_{i}(\omega) x \geq 0, \forall i \in \mathbf{N}\right\} . \\
& \text { imsart ver. 2011/05/20 file: Essup-22-06.tex date: June 22, } 2012
\end{aligned}
$$


From now on we suppose that the values of $G$ are proper cones, i.e. $G \cap(-G)=\{0\}$ a.s. (or, equivalently, int $G^{*} \neq \emptyset$ ). In the terminology of mathematical finance this property is called the efficient friction condition.

Under the adopted hypothesis the relation $\gamma_{2}-\gamma_{1} \in G$ a.s. defines a partial order $\gamma_{2} \succeq \gamma_{1}$ in $L^{0}\left(\mathbf{R}^{d}, \mathcal{F}\right)$. Moreover, the countable family of functions $u_{j}(\omega, x)=\eta_{j}(\omega) x$ where $\eta_{j}$ is a Castaing representation of $G^{*}$, represents the partial order defined by $G$. So, the above theory can be applied.

Notation. Let $\mathcal{H}$ is a sub- $\sigma$-algebra of $\mathcal{F}$ and let $\Gamma \subseteq L^{0}\left(\mathbf{R}^{d}, \mathcal{F}\right)$. We shall use sometimes the notation $(\mathcal{H}, G)$-Esssup $\Gamma$ instead of $\mathcal{H}$-Esssup $\Gamma$ to indicate that partial order is generated by the random cone $G$.

Note that in some cases we may dispose an additional information about the measurability of $G$.

Of course, if the partial order is given by a countable family of functions $(\omega, x) \mapsto \eta_{i}(\omega) x$, then we can generate it by a random cone $G$ given by (4.1). It is worth noting that in applications the partial order is usually given by a random cone rather than by a representing family.

Since in the considered case the order intervals $\left[\gamma_{1}(\omega), \gamma_{2}(\omega)\right]$ are compacts, the set $(\mathcal{H}, G)$-Esssup $\Gamma$ exists if $\Gamma$ is bounded from above with respect to the partial order (i.e. there is $\bar{\gamma} \in L^{0}\left(\mathbf{R}^{d}, \mathcal{H}\right)$ such that $\left.\bar{\gamma}-\Gamma \in G\right)$.

\subsection{Properties.}

In the following the partial order $\succeq$ is defined by the random cone $G$.

LEMma 4.1. Suppose that the representation (4.1) is given by d elements $\eta_{i} \in L^{0}\left(G^{*}, \mathcal{H}\right)$ such that the vectors $\eta_{i}(\omega)$ form a basis in $\mathbf{R}^{d}$ for almost all $\omega$ and $G^{*}=\operatorname{cone}\left(\eta_{i}\right)_{1 \leq i \leq d}$. Let $\Gamma \subseteq L^{0}\left(\mathbf{R}^{d}, \mathcal{F}\right)$ be such that $(\mathcal{H}, G)-\operatorname{Esssup} \Gamma$ is a singleton $\{\bar{\gamma}\}$. Then $\eta_{i} \bar{\gamma}=\left(\mathcal{H}, \mathbf{R}_{+}\right)-\operatorname{Esssup}\left(\eta_{i} \Gamma\right)$ for all $i \leq d$.

Proof. Let us consider $\eta:=\eta_{i}$ for some $i=1, \cdots, d$. Since $\bar{\gamma} \succeq \Gamma$, we have the inequalities $\eta \bar{\gamma} \geq \eta \gamma$ for all $\gamma \in \Gamma$. Therefore, $\eta \bar{\gamma} \geq\left(\mathcal{H}, \mathbf{R}_{+}\right)$-Esssup $(\eta \Gamma)$. Suppose that the inequality above is strict on a non-null set. Without loss of generality we may assume that $\eta_{1}=\eta$. Let us consider a $\mathcal{H}$-measurable random vector $\xi$ with $|\xi|=1$ such that $\xi(\omega)$ is orthogonal to the linear subspace generated by $\eta_{i}(\omega), i=2, \ldots, d$, for almost all $\omega$. We may always assume that $\eta \xi \geq 0$. We can find a non-zero $\mathcal{H}$-measurable random variable $\alpha \geq 0$ such that

$$
\eta(\bar{\gamma}-\alpha \xi) \geq\left(\mathcal{H}, \mathbf{R}_{+}\right)-\operatorname{Esssup}(\eta \Gamma) .
$$

It follows that $\bar{\gamma}-\alpha \xi \succeq \Gamma$ and, hence $\bar{\gamma}-\alpha \xi \succeq \bar{\gamma}$. Thus, we have that 
$\xi(\omega) \in G(\omega) \cap(-G(\omega))$ on the non-null set where $\alpha \neq 0$, i.e. $\xi(\omega)=0$ on this set. This is a contradiction.

Corollary 4.2. Assume that there are $d$ elements $\eta_{i} \in L^{0}\left(G^{*}, \mathcal{H}\right)$ such that the vectors $\eta_{i}(\omega)$ form a basis in $\mathbf{R}^{d}$ a.s. and $G^{*}=\operatorname{cone}\left(\eta_{i}\right)_{1 \leq i \leq d}$. Suppose that $(\mathcal{H}, G)$-Esssup of any finite subset of $L^{0}\left(\mathbf{R}^{d}, \mathcal{F}\right)$ is a singleton. Let $\Gamma \subseteq L^{0}\left(\mathbf{R}^{d}, \mathcal{F}\right)$ and let $(\mathcal{H}, G)$-Esssup $\Gamma=\{\hat{\gamma}\}$. Then for any $\eta \in L^{0}\left(\mathbf{R}^{d}, \mathcal{H}\right)$ there exists a sequence $\gamma_{n}$ from the set $\Gamma^{\mathrm{up}}$ (defined by (3.1)) such that

$$
\eta \hat{\gamma}=\lim _{n} \eta \gamma_{n}
$$

In particular, if $\Gamma=\Gamma^{\mathrm{up}}$, then $\eta \hat{\gamma} \leq\left(\mathcal{H}, \mathbf{R}_{+}\right)-\operatorname{esssup}(\eta \Gamma)$.

Proof. By virtue of Lemma 3.6, we may assume without loss of generality that $\Gamma=\Gamma^{\text {up }}$, i.e. $\Gamma$ is directed upwards. Since the vectors $\eta_{j}(\omega)$ form a basis in $\mathbf{R}^{d}, \eta=\sum_{j \leq d} \alpha_{j} \eta_{j}$ where $\alpha_{j} \in L^{0}(\mathbf{R}, \mathcal{H})$. By virtue of the above lemma,

$$
\eta \hat{\gamma}=\sum_{j \leq d} \alpha_{j}\left(\mathcal{H}, \mathbf{R}_{+}\right)-\operatorname{esssup}\left(\eta_{j} \Gamma\right)
$$

For each $j$, the family $\eta_{j} \Gamma$ is directed upwards. Thus, there are sequences $\gamma_{n}^{j} \in \Gamma$ such that $\eta_{j} \gamma_{n}^{j} \uparrow\left(\mathcal{H}, \mathbf{R}_{+}\right)$-esssup $\left(\eta_{j} \Gamma\right)$ a.s. Replacing the sequences $\left(\gamma_{n}^{j}\right)$ by the sequence $\gamma_{n}:=(\mathcal{H}, G)$-esssup $\left\{\gamma_{n}^{j}: j \leq d\right\} \in \Gamma$, we obtain that $\left(\mathcal{H}, \mathbf{R}_{+}\right)-\operatorname{esssup}\left(\eta_{j} \Gamma\right)=\lim _{n} \eta_{j} \gamma_{n}$. The statement follows from here immediately.

\subsection{Polyhedral Ordering Cones $G$ with Linearly Independent Generators.}

Proposition 4.3. Let $G=$ cone $\left\{\xi^{i}, i=1, \cdots, N\right\}$ where $\xi^{i} \in L^{0}\left(\mathbf{R}^{d}, \mathcal{F}\right)$ and, for every $\omega$, the vectors $\xi^{i}(\omega), i=1, \cdots, N$, are linearly independent (so, $N \leq d)$. Let a non-empty set $\Gamma \subseteq L^{0}\left(\mathbf{R}^{d}, \mathcal{F}\right)$ be such that $\Gamma \preceq \bar{\gamma}$ for some $\bar{\gamma} \in L^{0}\left(\mathbf{R}^{d}, \mathcal{F}\right)$. Then $\mathcal{F}$-Essup $\Gamma$ is a singleton.

Proof. Without loss of generality we may assume that $\left|\xi^{i}\right|=1$. Let us consider a $\mathcal{F}$-measurable random linear subspace $G-G$. Any $\gamma \in L^{0}(G-G, \mathcal{F})$ admits a unique representation $\gamma=\sum_{i=1}^{N} \alpha^{i}(\gamma) \xi^{i}$ where the coefficients $\alpha^{i} \in L^{0}(\mathbf{R}, \mathcal{F})$; they are all non-negative if and only if $\gamma \in L^{0}(G, \mathcal{F})$. Invariance under a shift on a fixed random vector allows us to reduce the problem to the case where $0 \in \Gamma$. Since $\bar{\gamma}-\Gamma \subset L^{0}(G, \mathcal{F})$, the "bound" $\bar{\gamma} \in L^{0}(G, \mathcal{F})$ and $\Gamma \subset L^{0}(G-G, \mathcal{F})$. Then

$$
\begin{aligned}
& \widehat{\alpha}_{i}:=\left(\mathbf{R}_{+}, \mathcal{F}\right) \text {-esssup }\left\{\alpha^{i}(\gamma), \gamma \in \Gamma\right\} \leq \alpha_{i}(\bar{\gamma})<\infty \\
& \text { imsart ver. 2011/05/20 file: Essup-22-06.tex date: June 22, } 2012
\end{aligned}
$$


(this is nothing but the classical essential supremum). It is easy to check that $\mathcal{F}$-Essup $\Gamma=\{\widehat{\gamma}\}$ where $\widehat{\gamma}=\sum_{i=1}^{N} \widehat{\alpha}^{i} \xi^{i}$.

Corollary 4.4. Under the assumptions of the above proposition on $G$, if a non-empty set $\Gamma \subseteq L^{0}\left(\mathbf{R}^{d}, \mathcal{F}\right)$ is such that $\left(\mathbf{R}_{+}, \mathcal{F}\right)$-esssup $|\Gamma|<\infty$, then Essup $\Gamma$ is a singleton.

\section{Hedging of European Options in a Discrete-Time Model with Transaction Costs.}

In the model we are given a closed proper convex cone $K \subset \mathbf{R}^{d}$ which interior contains $\mathbf{R}_{+}^{d} \backslash\{0\}$ and a stochastic basis $\left(\Omega, \mathcal{F}, \mathbf{F}=\left(\mathcal{F}_{t}\right)_{t=0, \ldots, T}, P\right)$ with a $d$-dimensional adapted process $S=\left(S_{t}\right)$ with strictly positive components.

Define the random diagonal operators

$$
\phi_{t}:\left(x^{1}, \ldots, x^{d}\right) \mapsto\left(x^{1} / S_{t}^{1}, \ldots, x^{d} / S_{t}^{d}\right), \quad t=0, \ldots, T,
$$

and relate with them the random cones $\widehat{K}_{t}:=\phi_{t} K$. We consider the set $\widehat{\mathcal{V}}$ of $\mathbf{R}^{d}$-valued adapted processes $\widehat{V}$ such that $\Delta \widehat{V}_{t}:=\widehat{V}_{t}-\widehat{V}_{t-1} \in-\widehat{K}_{t}$ for all $t$ and the set $\mathcal{V}$ which elements are the processes $V$ with $V_{t}=\phi_{t}^{-1} \widehat{V}_{t}, \widehat{V} \in \widehat{\mathcal{V}}$.

In the context of the theory of markets with transaction costs, $K$ is the solvency cone corresponding to the description of the model in terms of a numéraire, $\mathcal{V}$ is the set of value processes of self-financing portfolios. The notations with hat correspond to the description of the model in terms of "physical" units where the portfolio dynamics is much simpler because it does not depend on price movements. A typical example is the model of currency market defined via the matrix of transaction costs coefficients $\Lambda=\left(\lambda^{i j}\right)$. In this case

$$
K=\text { cone }\left\{\left(1+\lambda^{i j}\right) e_{i}-e_{j}, e_{i}, 1 \leq i, j \leq d\right\} .
$$

Note that we consider that here the matrix of transaction costs is assumed to be constant.

In this model the contingent claim is a $d$-dimensional random vector. We shall use the notation $Y_{T}$ when the contingent claim is expressed in units of the numéraire and $\widehat{Y}_{T}$ when it is expressed in physical units. The relation is obvious: $\widehat{Y}_{T}=\phi_{T} Y_{T}$.

The value process $V \in \mathcal{V}$ is called minimal if $V_{T}=Y_{T}$ and any process $W \in \mathcal{V}$ such that $W_{T}=Y_{T}$ and $W_{t} \preceq_{K} V_{t}$ for all $t \leq T$ coincides with $V$. The questions of interest are whether minimal portfolios do exist and how they can be found. We denote $\mathcal{V}_{\text {min }}$ the set of all minimal processes. The set $\widehat{\mathcal{V}}_{\text {min }}$ is defined in the obvious way.

imsart ver. 2011/05/20 file: Essup-22-06.tex date: June 22, 2012 
Proposition 5.1. Suppose that $L^{0}\left(\widehat{K}_{t+1}, \mathcal{F}_{t}\right) \subseteq L^{0}\left(\widehat{K}_{t}, \mathcal{F}_{t}\right), t \leq T-1$ and suppose there exits a least one $\widehat{V} \in \widehat{\mathcal{V}}$ such that $\widehat{V}_{T} \geq_{\hat{K}_{T}} \hat{Y}_{T}$. Then $\widehat{\mathcal{V}}_{\text {min }} \neq \emptyset$ and $\widehat{\mathcal{V}}_{\text {min }}$ coincides with the set of solutions of backward inclusions

$$
\widehat{V}_{t} \in\left(\mathcal{F}_{t}, \widehat{K}_{t+1}\right)-\operatorname{Esssup}\left\{\widehat{V}_{t+1}\right\}, \quad t \leq T-1, \quad \widehat{V}_{T}=\widehat{Y}_{T} .
$$

Moreover, any $W \in \mathcal{V}$ with $W_{T} \succeq Y_{T}$ is such that $W \succeq_{K} V$ for some $V \in \mathcal{V}_{\min }$.

Proof. Let $\widehat{W} \in \widehat{\mathcal{V}}$ be such that $\widehat{W}_{T} \succeq_{\widehat{K}_{T}} \widehat{Y}_{T}$. Since $\Delta \widehat{W}_{T} \in-\widehat{K}_{T}$, we have $\widehat{W}_{T-1} \succeq_{\widehat{K}_{T}} \widehat{W}_{T}$. By definition of $\left(\mathcal{F}_{T-1}, \widehat{K}_{T}\right)$-Esssup and Theorem 3.7, we obtain that $\widehat{W}_{T-1} \succeq_{\widehat{K}_{T}} \widehat{V}_{T-1}$ for some $\widehat{V}_{T-1} \in\left(\mathcal{F}_{T-1}, \widehat{K}_{T}\right)$-Esssup $\left\{\widehat{Y}_{T}\right\} \neq \emptyset$. Therefore, by the hypothesis, $\widehat{W}_{T-1} \succeq_{\widehat{K}_{T-1}} \widehat{V}_{T-1}$. Continuing the backward induction, we obtain that $\widehat{W}_{t} \succeq_{\widehat{K}_{t}} \widehat{V}_{t}$ where $\widehat{V}_{t}$ satisfies (5.1). We deduce that any portfolio $\widehat{W} \in \widehat{\mathcal{V}}_{\text {min }}$ satisfy (5.1). The same backward induction allows us to conclude that any $\widehat{V} \in \widehat{\mathcal{V}}$ which satisfies (5.1) is minimal.

REMARK 5.2. The hypothesis $L^{0}\left(\widehat{K}_{t+1}, \mathcal{F}_{t}\right) \subseteq L^{0}\left(\widehat{K}_{t}, \mathcal{F}_{t}\right), t \leq T-1$, of the above proposition is equivalent to the absence of arbitrage opportunities of the second kind, see [2], Th. 3.2.20. Note that it is always fulfilled when the price process $S$ admits an equivalent martingale measure. This hypothesis is essential for the claimed property. Indeed, with $T=1$, suppose that the inclusion $L^{0}\left(\widehat{K}_{1}, \mathcal{F}_{0}\right) \subseteq L^{0}\left(\widehat{K}_{0}, \mathcal{F}_{0}\right)$ does not hold. In this case, we may find $\hat{W}_{0} \in L^{0}\left(\widehat{K}_{1}, \mathcal{F}_{0}\right)$ such that $\hat{W}_{0} \notin \widehat{K}_{0}$. With the payoff $\hat{Y}_{T}=0$, we easily obtain that the only minimal value process which satisfies (5.1) is given by $\hat{V}_{1}=\hat{V}_{0}=0$. If the assertion of Proposition 5.1 holds, the value process $\left(\hat{W}_{0}\right.$, $\left.\hat{W}_{1}=0\right)$ should satisfy $\hat{W}_{0} \succeq \hat{V}_{0}$, i.e. $\hat{W}_{0} \in \hat{K}_{0}$ and we get a contradiction.

REMARK 5.3. In general, the essential supremum in the formula (5.1) is not a singleton. Take for instance the simple case where $\Omega:=\left\{\omega_{1}, \omega_{2}\right\}$ and $T=1$. Consider $\mathcal{F}_{0}:=\{\emptyset, \Omega\}$ and $\mathcal{F}_{1}$ is the $\sigma$-algebra of all subsets of $\Omega$. Suppose that $\widehat{K}_{0}=\widehat{K}_{1}\left(\omega_{1}\right)$ and $\widehat{K}_{1}\left(\omega_{2}\right) \neq \widehat{K}_{0}$. As illustrated in the picture below, if $\widehat{V}_{1}=A I_{\left\{\omega_{1}\right\}}+B I_{\left\{\omega_{2}\right\}}$, then $\left(\mathcal{F}_{0}, \widehat{K}_{1}\right)$-Esssup $\left\{\widehat{V}_{1}\right\}=\Lambda$ where $\Lambda:=\left(A+\widehat{K}_{0}\right) \cap\left(B+\widehat{K}_{1}\left(\omega_{2}\right)\right)$. Indeed, the set of all deterministic points $V_{0} \geq \widehat{K}_{1} \widehat{V}_{1}$ is $\Lambda$ and neither of them can be "reduced" in the direction of $\widehat{K}_{1}$ since $\widehat{K}_{1}\left(\omega_{2}\right) \cap \widehat{K}_{1}\left(\omega_{1}\right)=\{0\}$. 


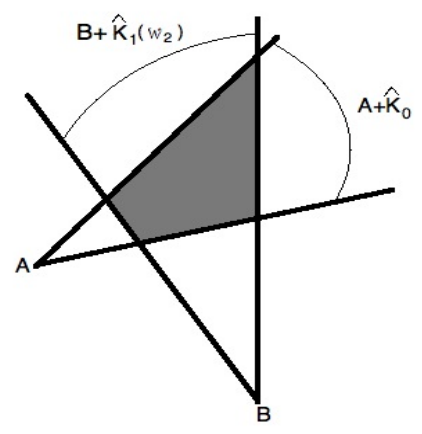

FIG 2. The grey-coloured domain corresponds to the set $\Lambda:=\left(\mathcal{F}_{0}, \widehat{K}_{1}\right)$-Esssup $\left\{\widehat{V}_{1}\right\}$ where $\widehat{V}_{1}=A I_{\left\{\omega_{1}\right\}}+B I_{\left\{\omega_{2}\right\}}$.

\section{References.}

[1] Evren O., Ok E.A. On the multi-utility representation of preference relations. Journal of Mathematical Economics, 14 (2011), 4-5, 554-563.

[2] Kabanov Yu., Safarian M. Markets with Transaction Costs. Mathematical Theory. Springer-Verlag, 2009.

[3] Löhne A. Vector Optimization with Infimum and Supremum. Springer-Verlag.

[4] Molchanov I. Theory of Random Sets. Springer-Verlag, 2005.

Laboratoire de Mathématiques, Université de Franche-Comté, 16 Route de Gray, 25030 Besançon cedex, France, and

Institute of Informatics Problems of the Russian Academy of Sciences, Moscow, Russia.

Ceremade, Place du Maréchal De Lattre De Tassigny,

75775 Paris Cedex 16, France

E-MAIL: youri.kabanov@univ-fcomte.fr; emmanuel.lepinette@ceremade.dauphine.fr 